\title{
Leveraging Conditional Linkage Models in Gray-box Optimization with the Real-Valued Gene-pool Optimal Mixing Evolutionary Algorithm
}

\author{
Supplementary Material \\ Anton Bouter \\ Centrum Wiskunde \& Informatica \\ Amsterdam, The Netherlands \\ Anton.Bouter@cwi.nl \\ Tanja Alderliesten \\ Leiden University Medical Center \\ Leiden, The Netherlands \\ T.Alderliesten@lumc.nl \\ Stefanus C. Maree \\ Amsterdam UMC \\ University of Amsterdam \\ Amsterdam, The Netherlands \\ S.C.Maree@amsterdamumc.nl \\ Peter A.N. Bosman \\ Centrum Wiskunde \& Informatica \\ Amsterdam, The Netherlands \\ Delft University of Technology \\ Delft, The Netherlands \\ Peter.Bosman@cwi.nl
}

\section{CCS CONCEPTS}

- Mathematics of computing $\rightarrow$ Evolutionary algorithms;

\section{ACM Reference Format:}

Anton Bouter, Stefanus C. Maree, Tanja Alderliesten, and Peter A.N. Bosman. 2020. Leveraging Conditional Linkage Models in Gray-box Optimization with the Real-Valued Gene-pool Optimal Mixing Evolutionary Algorithm: Supplementary Material. In Proceedings of the Genetic and Evolutionary Computation Conference 2020 (GECCO '20). ACM, New York, NY, USA, 2 pages. https://doi.org/10.1145/nnnnnnn.nnnnnnn

\section{A FREQUENCY OF SELECTION IN RV-GOMEA}

In this section we show how applying selection (and an update of the sampling relevant sampling distributions) at the start of each iteration of GOM affects the performance of RV-GOMEA [1], compared to previous experiments. We compare the originally used generational selection (Gen.Sel.) to selection applied at the start of each iteration of GOM (GOM.Sel.).

The sphere, Rosenbrock and SoREB benchmark problems are considered, as in [1]. For each benchmark problem, two reasonable linkage models are considered. Results for these experiments are shown in Figure 1. Medians of 30 runs are shown, along with error bars displaying 10th and 90th percentiles. Population sizes were determined by running a bisection.

As expected, we observe no difference between the two selection methods for the marginal product linkage models (Univariate and MPM5). Minor differences in performance are observed for the Bounded Fixed Linkage Tree (BFLT) model, most notable on the Rosenbrock problem, for which selection at the start of each

Permission to make digital or hard copies of part or all of this work for personal or classroom use is granted without fee provided that copies are not made or distributed for profit or commercial advantage and that copies bear this notice and the full citation on the first page. Copyrights for third-party components of this work must be honored.

For all other uses, contact the owner/author(s).

GECCO '20, July 8-12, 2020, CancÃžn, Mexico

(ㄷ) 2020 Copyright held by the owner/author(s).

ACM ISBN 978-x-xxxx-xxxx-x/YY/MM...\$15.00

https://doi.org/10.1145/nnnnnnn.nnnnnnn iteration of GOM was favored. Note that large error bars on the Rosenbrock problem were the effect of runs converged to the local minimum, as no restart mechanism was used.

Based on the results shown in Figure 1, we argue that selection at the start of each iteration of GOM has no negative effect on the performance of RV-GOMEA on previously considered benchmark problems. As argued in the main article, we therefore employ this form of selection, as it benefits the application of conditional linkage models to RV-GOMEA.

\section{REFERENCES}

[1] A. Bouter, T. Alderliesten, C. Witteveen, and P.A.N. Bosman. 2017. Exploiting linkage information in real-valued optimization with the real-valued gene-pool optimal mixing evolutionary algorithm. In Proceedings of the Genetic and Evolutionary Computation Conference. ACM, 705-712. 

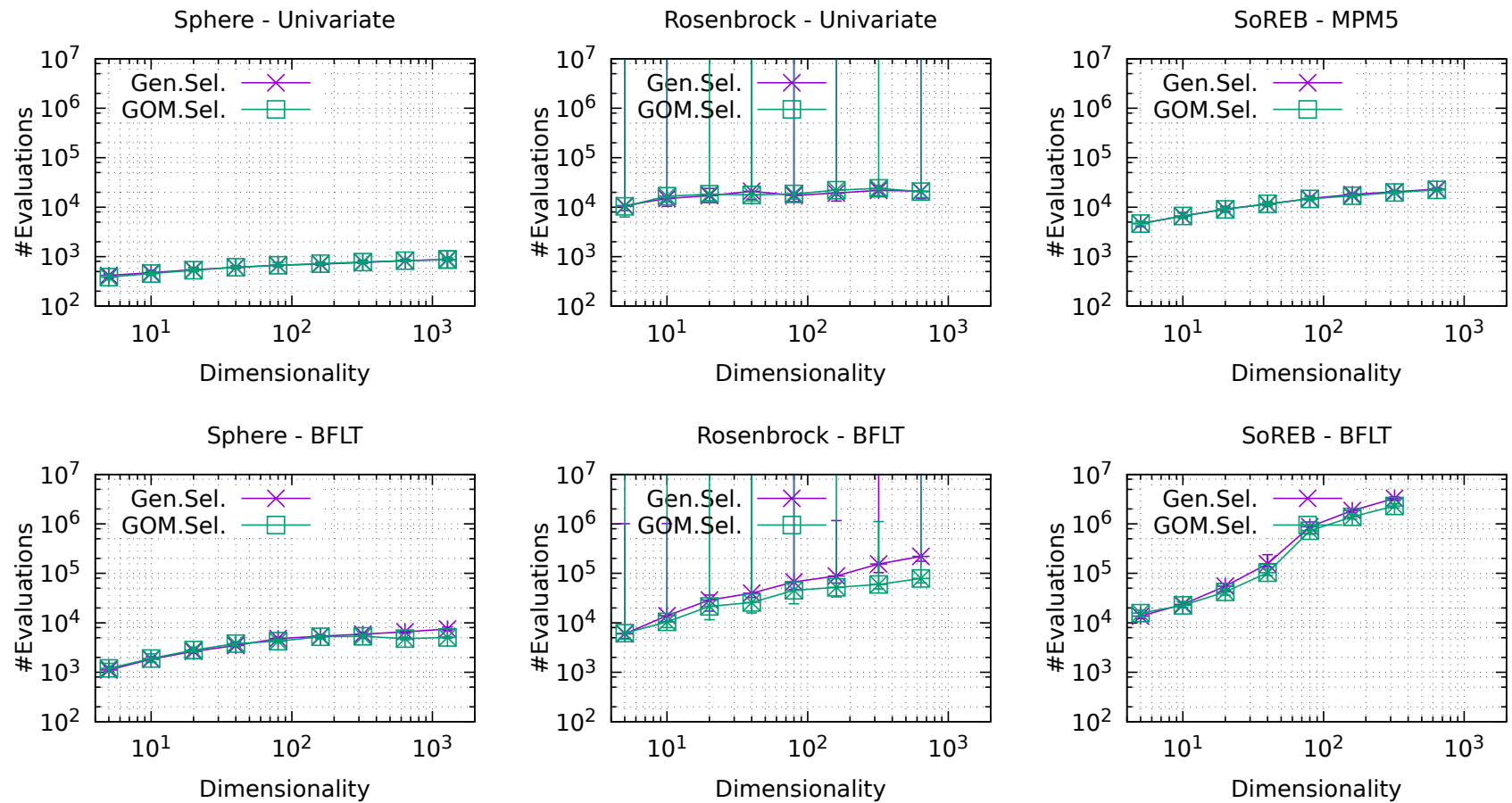

Figure 1: Median, 10th percentile and 90th percentile (30 runs) number of evaluations for various linkage models for various benchmark problems in a GBO setting. Selection was either used at the start of each generation (Gen.Sel.), or at the start of each iteration of GOM (GOM.Sel.). 